\title{
Prevalência do consumo moderado e excessivo de álcool e fatores associados entre residentes de Comunidades Quilombolas de Vitória da Conquista, Bahia, Brasil
}

\author{
Prevalence of moderate and excessive alcohol consumption \\ and associated factors among residents of Quilombo Communities \\ (hinterland settlements founded by people of African origin) \\ of Vitória da Conquista, Bahia, Brazil
}

Luiz Gustavo Vieira Cardoso ${ }^{1}$

Ana Paula Souto Melo ${ }^{2}$

Cibele Comini Cesar ${ }^{3}$

${ }^{1}$ Instituto Multidisciplinar em Saúde, Universidade Federal da Bahia. Av. Olivia Flores, Universitário. 45031-000 Vitória da Conquista BA Brasil. luizgvc11@gmail.com

${ }^{2}$ Faculdade de Medicina, Universidade Federal de São João Del-Rei.

${ }^{3}$ Departamento de Estatística, Universidade Federal de Minas Gerais.

\begin{abstract}
The scope of the study was to assess moderate and excessive alcohol consumption and associated factors among residents of Quilombola Communities in Vitória da Conquista in the State of Bahia. It involved a cross-sectional study among 750 individuals aged $\geq 18$ years interviewed in 2011 using an adapted version of the National Survey of Health Questionnaire. Alcohol consumption was classified into abstemious, moderate and heavy drinking categories and was analyzed using the multinomial regression model. Consumption was observed among $41.5 \%$ of participants (95\% CI [37.8, 44.98]); 10.7\% being identified in the excessive consumption category and $30.8 \%$ in the moderate consumption category. Moderate consumption was inversely associated with age and is more common among whites and mulattoes, the better educated, the gainfully employed and among smokers. Heavy consumption increased with level of education, being more prevalent among those who had at least 8 years of schooling, smokers and the gainfully employed. The study shows that in these communities there are differences in the sociodemographic characteristics among the profiles of alcohol drinkers and these differences should be taken into account when drawing up proposals for the promotion of healthy habits.
\end{abstract}

Key words Alcohol consumption, Quilombola communities, Sociodemographic characteristics
Resumo O objetivo do presente estudo foi analisar o consumo, seja moderado ou excessivo, de álcool entre residentes de comunidades quilombolas de Vitória da Conquista (BA), bem como os fatores associados. Estudo transversal envolvendo 750 indivíduos com idade $\geq 18$ anos, entrevistados em 2011, utilizando uma versão adaptada do questionário da Pesquisa Nacional de Saúde. O consumo de bebidas alcoólicas foi classificado em abstenção, uso moderado ou excessivo, e analisado por meio do modelo de regressão multinomial. Observou-se o consumo entre $41,5 \%$ dos participantes (IC95\%\%: [37,8; 44,98]); sendo identificados na categoria consumo excessivo e, na categoria consumo moderado, 10,7\% e 30,8\%, respectivamente. O consumo moderado estava relacionado inversamente com a idade, sendo mais frequente entre individuos brancos ou pardos, os mais escolarizados, aqueles em atividade laborativa e entre fumantes. O consumo excessivo aumentou com o grau de escolaridade, sendo mais prevalente entre os que tinham pelo menos 8 anos de estudo, os fumantes e os que exerciam atividade laborativa. O estudo mostra que nessas comunidades existem diferenças segundo as características sociodemográficas dos diversos perfis de consumidores de álcool, e que estas diferenças devem ser consideradas na elaboração de propostas para promoção de hábitos saudáveis.

Palavras-Chave Consumo de álcool, Comunidades quilombolas, Características sociodemográficas 


\section{Introdução}

O álcool é uma das poucas substâncias psicoativas que tem seu consumo admitido pela sociedade $^{1}$ e seu uso sempre fez parte da história da humanidade. Quando utilizado excessivamente, entretanto, está associado a problemas sociais e de comportamento e a um grande número de agravos à saúde $\mathrm{e}^{1-3}$. A World Healh Organization $(\mathrm{WHO})^{4}$ menciona que a população brasileira encontra-se entre os maiores consumidores mundiais de álcool. Nesse sentido, a política brasileira vem propondo ações de promoção da saúde com ênfase em ações educativas e veiculação de informações sobre os danos do uso abusivo do álcool, além de propor iniciativas para a restrição de acesso a bebidas alcoólicas pelos segmentos vulneráveis ${ }^{5,6}$.

A identificação epidemiológica dos segmentos sociodemográficos mais susceptíveis ao consumo e à dependência alcoólica é fundamental para avaliar programas e orientar estratégias mais efetivas de controle e redução de danos. As desvantagens socioeconômicas associadas a fatores individuais aumentam a probabilidade de início precoce do consumo de bebidas alcoólicas e de mudanças do padrão de consumo do álcool, bem como de iniciação ao consumo de outras substâncias psicoativas 5 , 7.8 .

Estudo com a população brasileira já demonstra que o consumo moderado (ingestão de uma dose/dia para as mulheres e duas doses/dia para os homens) de álcool associa-se à melhor situação socioeconômica, nível de escolaridade mais elevado e inserção no mercado de trabalho ${ }^{1}$.

No Brasil, existem particularidades referentes ao consumo excessivo de álcool. Nas regiões sul e sudeste do país identificam-se aumento de consumo excessivo entre pessoas do sexo masculino, de cor de pele negra ou parda, não casadas, sem religião, de nível social mais baixo e tabagistas ${ }^{3,9}$. Em estudos realizados na região nordeste não se verificou associação entre o padrão de consumo e a cor da pele en-12. $^{10}$.

A despeito das limitações desse indicativo, a cor da pele pode captar as desigualdades em saúde às quais grupos sociais como os quilombolas estão expostos ${ }^{13,14}$. Estes grupos sociais se distinguem dos demais pela identidade étnica através de critérios de autoatribuição e trajetória histórica própria, tendo práticas de manutenção e reprodução de modos de vida característicos, com presunção de ancestralidade negra relacionada com a resistência à opressão histórica sofrida ${ }^{15}$.

Araújo et al. ${ }^{14}$ apontam que a população negra do Brasil ocupa posições menos qualificadas e de pior remuneração no mercado de trabalho; reside em áreas com ausência ou baixa disponibilidade de serviços de infraestrutura básica; sofre maiores restrições no acesso a serviços de saúde e, estes, quando disponibilizados, são de pior qualidade e menor resolutividade.

É nesse contexto que pesquisas recentes vêm incorporando o estudo das iniquidades sociais como um dos determinantes das condições de saúde ${ }^{16}$, entre os quais as iniquidades étnico-raciais emergem como foco de investigações. Este enfoque se justifica dada a persistência de disparidades raciais, evidenciadas nos negros, que indicam maior incidência de doenças e por morrerem mais precocemente, em todas as idades ${ }^{17}$.

Nesse sentido, produções acadêmicas problematizam a "saúde da população negra", relacionando os conceitos de vulnerabilidade e racismo e seus impactos na saúde ${ }^{13,18}$. Assim, a discussão de questões relacionados ao uso do álcool em populações vulneráveis como rurais, indígenas e quilombolas são de extrema relevância considerando o contexto social, cultural e histórico específico, além da organização social destes povos e sua história de contato com a sociedade $e^{19,20}$.

Estudos identificando padrões de consumo de bebidas alcoólicas que ajudem na compreensão da magnitude do problema em grupos populacionais específicos, como os quilombolas, são necessários para fornecer informações a gestores de saúde a fim de que se possam desenvolver ações e políticas eficazes de prevenção e de controle do abuso de álcool ${ }^{4}$.

Analisando as características geográficas das comunidades quilombolas, geralmente localizadas em áreas rurais, com um relativo grau de isolamento geográfico atribuído à decadência das atividades agrícolas, nota-se que a compreensão do processo de territorialização é necessária, pois a maioria das comunidades foi formada a partir de quilombos, por constituir-se em uma manifestação de insubordinação ao modelo escravocrata. Amorin e Germani ${ }^{21}$ supõem que, a partir da distribuição espacial dos territórios quilombolas no país, alguns fatores como dificuldade de acesso, recursos naturais, relações de permissividade, perante grupos que hegemonizam o poder local, articulação com setores da sociedade circundante, contribuíram para determinar a localização dessas comunidades, principalmente na faixa litorânea e região Nordeste. Por isso, estes territórios de risco possuem a necessidade de mensurar os quesitos relacionados ao consumo de álcool em inquéritos de saúde, pois tais populações são vítimas de iniquidades territoriais, em saúde e também no acesso aos serviços de saúde ${ }^{22}$. 
A compreensão dos fatores associados com o consumo de álcool é aspecto importante para a eficácia das políticas e a prevenção dos danos associados ao uso dessa substância ${ }^{5}$. Diante do exposto, o objetivo do presente estudo é avaliar o consumo moderado e excessivo de álcool e identificar fatores associados entre residentes de comunidades quilombolas do município de vitória da Conquista, Bahia.

\section{Material e método}

\section{Características da Amostra}

Estudo de corte transversal realizado em comunidades quilombolas, localizadas no município de Vitória da Conquista-Ba, no período de setembro a outubro de 2011. Parte integrante do projeto COMQUISTA: Comunidades Quilombolas de Vitória da Conquista (BA): Avaliação de saúde e seus condicionantes, realizado pela Universidade Federal da Bahia (UFBA) e Universidade Federal de Minas Gerais (UFMG).

O município de Vitória da Conquista, localizado no Sudoeste da Bahia, dista $509 \mathrm{~km}$ da capital Salvador, com 320.129 habitantes, possui 11 distritos dos quais 5 distritos possuem comunidades quilombolas certificadas pela Fundação Cultural Palmares, segundo dados do Sistema de Informação da Atenção Básica (SIAB) fornecidos pela Secretaria Municipal da Saúde. No total existem 25 comunidades quilombolas no local, que distam em média 31,2 km da sede do município.

A população alvo foi composta por indivíduos adultos ( $\geq 18$ anos) residentes nas comunidades quilombolas com pelo menos 50 famílias, localizadas na zona rural do município e foi estimada em 2.935 indivíduos. O cálculo do tamanho amostral baseou-se nos seguintes parâmetros: prevalência de $50 \%$, precisão de $5 \%$, intervalo de confiança de $95 \%$ e efeito de desenho = 2 , que levaram a uma estimativa de tamanho de amostra inicial de 649 indivíduos. Considerou-se a possibilidade de ocorrência de $30 \%$ de perdas, o que levou a um tamanho final de amostra de 844 indivíduos.

Dos cinco distritos que registraram a existência de comunidades quilombolas certificadas, dois tinham apenas uma comunidade quilombola e estas foram incluídas no estudo. Em cada um dos outros três distritos foi feita seleção aleatória de uma comunidade quilombola, com probabilidade proporcional ao número de habitantes da comunidade, totalizando cinco comunidades. Selecionadas as comunidades, procedeu-se a se- leção aleatória dos domicílios, com probabilidade proporcional ao número de domicílios do distrito. Todos os moradores adultos dos domicílios selecionados foram convidados a participar da pesquisa $^{23}$.

O projeto foi aprovado pelos Comitês de Ética em Pesquisa da Faculdade São Francisco de Barreiras e da Universidade Federal de Minas Gerais.

Neste estudo, foram excluídas mulheres em período gestacional, uma vez que a gestação altera o comportamento da mulher com relação ao uso do álcool e os indivíduos para os quais as informações foram relatadas por informantes secundários, considerando que a informação de consumo de substância psicoativa pode apresentar vício de declaração quando relatadas por informante secundário.

\section{Coleta de dados}

Os dados foram coletados por meio de entrevistas individuais, realizadas por entrevistadores treinados pela equipe coordenadora do projeto. O instrumento de entrevistas utilizado foi uma versão adaptada para a população Quilombola do questionário semiestruturado da Pesquisa Nacional de Saúde - $\mathrm{PNS}^{24}$. Este questionário foi previamente testado em estudo piloto realizado em uma comunidade quilombola similar não selecionada para a pesquisa ${ }^{23}$. As informações foram registradas em computador portátil (HP Pocket Rx5710), através do programa QDS Trial versão 2.6.1.

\section{Variáveis estudadas}

A variável resposta deste estudo foi o consumo de bebidas alcoólicas. Este foi caracterizado utilizando informações do questionário sobre padrão do uso regular de álcool (dias por semana), além da resposta à questão sobre seu uso excessivo em uma única ocasião (5 ou mais doses de bebida alcoólica em uma única ocasião para homens ou 4 doses ou mais para mulheres). $\mathrm{O}$ consumo foi classificado em três categorias - abstenção, uso moderado e uso excessivo -, considerando-se o número de doses consumidas semanalmente, cada dose correspondendo a cerca de 10-12g de álcool ${ }^{25}$. Foram classificados na categoria abstenção participantes que declararam não consumir álcool. O consumo moderado foi atribuído aos indivíduos que consomem até 15 doses/semana, se homem, ou 10 doses/semana, se mulher (consumo regular moderado), e não relataram ter consumido mais de 5 doses em uma 
única ocasião, se homem, ou 4 doses, se mulher. O consumo excessivo englobou o consumo regular excessivo e episódios de consumo excessivo, ou seja, consumo regular de mais de 15 doses por semana, se homem, ou mais de 10 doses, se mulher, ou consumo esporádico, em uma única ocasião, de, pelo menos, 5 doses, se homem, ou pelo menos 4 doses, se mulher ${ }^{5}$. O período considerado para avaliação do consumo excessivo esporádico foi de duas semanas.

As variáveis explicativas analisadas no estudo abrangeram as dimensões sociodemográficas, estilo de vida, condições de saúde e riscos associados ao consumo de álcool. As características sociodemográficas incluídas foram: autodefinição quilombola (sim, não), cor da pele autorreferida (preta, parda, branca/indígena/amarela); sexo (masculino, feminino); idade em grupo etário (18 a 24,25 a 34,35 a 44,45 a 59,60 e mais); escolaridade em anos completos de estudos agrupados ( 0,1 - 3, 4 - 7, 8 e mais); situação conjugal (com companheiro(a), sem companheiro(a); trabalha atualmente (sim, não). Os hábitos relacionados ao estilo de vida incluíam: prática regular de atividade física (sim, não); tabagismo atual (sim, não); consumo de frutas e verduras (5 ou mais dias na semana; menos de 5 dias na semana). Indicadoras das condições de saúde: auto avaliação de saúde (Muito Boa/Boa, Regular/ Ruim/Muito Ruim); história de Doença Crônica (Nenhuma, Uma, Duas ou mais). O número de doenças crônicas foi construído a partir das informações autorreferidas do diagnóstico médico de doze doenças crônicas (hipertensão arterial, diabetes, hipercolesterolemia, doença cardíaca, acidente vascular cerebral, asma ou bronquite, depressão, outra doença mental, doença pulmonar, artrite, problema na coluna e osteoporose - Não se considerou presença de hipertensão arterial ou diabetes exclusivamente durante gravidez); Além disso, foram verificados: abandono do trabalho por motivo de saúde (sim, não); depressão avaliada pela escala de triagem de depressão PHQ-9 e categorizado (sem depressão, deprimido); uso de medicamentos nos últimos 15 dias (sim, não). As variáveis de riscos associados ao consumo de bebidas alcoólicas foram: vítima de acidentes de trânsito (sim, não); vítimas de violência física /agressão (sim, não). As pessoas que se autodeclararam amarelas ou indígenas foram agrupadas com as brancas em uma única categoria, devido ao pequeno número de observações.

O uso do álcool entre adultos residentes nas comunidades quilombolas foi descrito através do cálculo da prevalência geral e estratificada por sexo com intervalo de confiança de $95 \%$ (IC95\%). As variáveis qualitativas nominais e ordinais foram descritas pela distribuição absoluta e percentual. Para verificar os fatores associados ao consumo moderado e excessivo de álcool utilizou-se o modelo logístico multinomial. Em um primeiro passo da análise foi estimado o efeito de cada variável explicativa sobre o consumo de bebidas alcoólicas. As variáveis significativas ao nível de $20 \%$ foram selecionadas para inclusão no modelo final, permanecendo aquelas variáveis significativas ao nível de significância de 5\%. Todos os modelos foram ajustados por idade e sexo. A comparação entre modelos foi feita pelo critério de Akaike (AIC) e a adequação do modelo final foi verificada pelo teste Hosmer-Lemeshow para modelo multinomial. As análises foram realizadas no programa Stata versão 12 (Stata Corporation, College Station, Estados Unidos), incorporando o efeito de delineamento da amostra. As variáveis de condições de saúde e de riscos associados ao consumo de álcool foram utilizadas apenas para descrição do perfil da população estudada, não sendo incorporadas nos modelos associativos pela baixa frequência dos eventos.

\section{Resultados}

Foram visitados 422 domićlios e identificados 943 indivíduos elegíveis para o estudo. Destes, 797 participaram da pesquisa. Perda de 13,3\% da amostra deveu-se, principalmente, ao não encontro dos indivíduos após três visitas ao domicílio. As perdas foram majoritariamente de indivíduos do sexo masculino $(77,4 \%)$ na faixa etária de 18 a 34 anos $(65,2 \%)$. A análise foi ajustada pelas perdas, através da incorporação dos pesos pós-estratificação.

Dos 797 indivíduos que concordaram em participar da pesquisa, foram excluídos desta análise 36 informantes secundários $(4,5 \%)$ e 11 gestantes $(1,4 \%)$, perfazendo uma amostra final de 750 indivíduos.

O consumo de bebidas alcoólicas foi declarado por $41,5 \%$ (IC95\%: [36,3; 51,7]) dos participantes. Considerando o perfil do consumo, 58,5\% (IC95\%: [55,0; 62,1]) dos indivíduos relataram ser abstêmios, 30,8\% (IC95\%: [27,5;34,1]) ter "Consumo moderado" e 10,7\% (IC95\%: [8,5; 12,9]) "Consumo excessivo". Dos que relataram consumo excessivo de álcool, 95\% relataram consumo excessivo esporádico, sendo que 27,6\% apresentavam também consumo excessivo regular. Somente 5\% foram classificados como con- 
sumidores excessivos por beberem regularmente em doses medianas.

A Tabela 1 apresenta a distribuição das variáveis explicativas para a amostra total e segundo as categorias do consumo de bebidas alcoólicas. Os participantes eram majoritariamente do sexo feminino $(53,5 \%)$, viviam com companheiro $(63,7 \%)$, da cor preta $(41,6 \%)$ ou parda $(44,0 \%)$, com autodeclaração quilombola $(83,2 \%)$, não chegaram a completar um ano na escola $(32,9 \%)$ e trabalhavam no momento da pesquisa $(51,2 \%)$.
A inatividade física foi relatada por $74,3 \%$ dos entrevistados, 19,7\% informaram ser tabagistas e $76,3 \%$ informaram consumo satisfatório de frutas, verduras e legumes.

A idade variou entre 18 e 100 anos com média de 44,0 (DP = 17,4). A idade mostrou comportamento diferencial segundo o padrão de consumo de álcool, com os abstêmios apresentando idade média de 46,9 anos ( $\mathrm{DP}=18,0)$ significativamente superior aos demais grupos: 39,9 $(\mathrm{DP}=15,7)$ para os consumidores moderados e

Tabela 1. Distribuição de frequência do consumo de bebidas alcoólicas moderado e excessivo em relação às variáveis demográficas, socioeconômicas e estilo de vida. Projeto COMQUISTA, Brasil, 2011.

\begin{tabular}{|c|c|c|c|c|}
\hline \multirow[b]{2}{*}{ Variáveis } & \multicolumn{4}{|c|}{ Consumo de bebidas alcoólicas } \\
\hline & $\begin{array}{l}\text { Amostra total } \\
\text { N (\%) }\end{array}$ & $\begin{array}{r}\text { Abstêmio } \\
\mathrm{N}(\%)\end{array}$ & $\begin{array}{c}\text { Moderado } \\
\text { N (\%) }\end{array}$ & $\begin{array}{c}\text { Excessivo } \\
\mathbf{N}(\%)\end{array}$ \\
\hline \multicolumn{5}{|l|}{ Idade } \\
\hline $18-24$ & $115(15,3)$ & $60(13,7)$ & $42(18,2)$ & $13(16,3)$ \\
\hline $25-34$ & $150(20,0)$ & $66(15,0)$ & $60(26,0)$ & $24(30,0)$ \\
\hline $35-44$ & $148(19,7)$ & $86(19,6)$ & $49(21,2)$ & $13(16,3)$ \\
\hline $45-59$ & $173(23,1)$ & $106(24,2)$ & $48(20,8)$ & $19(23,7)$ \\
\hline $60+$ & $164(21,9)$ & $121(27,6)$ & $32(13,8)$ & $11(13,7)$ \\
\hline \multicolumn{5}{|l|}{ Sexo } \\
\hline Feminino & $401(53,5)$ & $289(65,8)$ & $95(41,2)$ & $17(21,3)$ \\
\hline Masculino & $349(46,5)$ & $150(34,2)$ & $136(58,8)$ & $63(78,7)$ \\
\hline \multicolumn{5}{|l|}{ Situação Conjugal } \\
\hline Com companheiro(a) & $478(63,7)$ & $291(66,3)$ & $144(62,3)$ & $43(53,7)$ \\
\hline Sem companheiro(a) & $272(36,3)$ & $148(33,7)$ & $87(37,7)$ & $37(46,3)$ \\
\hline \multicolumn{5}{|l|}{ Cor da pele autorreferida } \\
\hline Preto & $312(41,6)$ & $192(43,7)$ & $89(38,5)$ & $31(38,7)$ \\
\hline Pardo & $330(44,0)$ & $190(43,3)$ & $102(44,2)$ & $38(47,5)$ \\
\hline Branco e outros & $108(14,4)$ & $57(13,0)$ & $40(17,3)$ & $11(13,8)$ \\
\hline \multicolumn{5}{|l|}{ Autodefinição quilombola } \\
\hline Não & $125(16,7)$ & $77(17,6)$ & $32(13,8)$ & $16(20,0)$ \\
\hline Sim & $624(83,2)$ & $361(82,4)$ & $199(86,2)$ & $64(80,0)$ \\
\hline \multicolumn{5}{|l|}{ Escolaridade } \\
\hline 0 & $247(32,9)$ & $173(39,4)$ & $53(22,9)$ & $21(26,2)$ \\
\hline $1-3$ & $209(27,9)$ & $122(27,8)$ & $70(30,3)$ & $17(21,3)$ \\
\hline $4-7$ & $206(27,5)$ & $111(25,3)$ & $67(29,0)$ & $28(35,0)$ \\
\hline 8 e mais & $88(11,7)$ & $33(7,5)$ & $41(17,8)$ & $14(17,5)$ \\
\hline \multicolumn{5}{|l|}{ Trabalha atualmente } \\
\hline Não & $366(48,8)$ & $271(74,0)$ & $78(21,3)$ & $17(4,7)$ \\
\hline Sim & $384(51,2)$ & $168(43,8)$ & $153(39,8)$ & $63(16,4)$ \\
\hline \multicolumn{5}{|l|}{ Tabagismo atual } \\
\hline Sim & $148(19,7)$ & $53(12,1)$ & $58(25,1)$ & $37(46,3)$ \\
\hline Não & $602(80,3)$ & $386(87,9)$ & $173(74,9)$ & $43(53,7)$ \\
\hline \multicolumn{5}{|c|}{ Prática regular de atividade física } \\
\hline Não & $557(74,3)$ & $347(79,1)$ & $158(68,4)$ & $52(65,0)$ \\
\hline Sim & $193(25,7)$ & $92(20,9)$ & $73(31,6)$ & $28(35,0)$ \\
\hline \multicolumn{5}{|c|}{ Consumo de verduras/legumes/frutas/suco } \\
\hline 5 vezes ou mais/semana & $572(76,3)$ & $327(74,5)$ & $183(79,2)$ & $62(77,5)$ \\
\hline$<5$ vezes/semana & $178(23,7)$ & $112(25,5)$ & $48(20,8)$ & $18(22,5)$ \\
\hline
\end{tabular}


$39,8(\mathrm{DP}=15,2)$ para os que consomem de maneira excessiva.

Homens e mulheres apresentaram diferença com relação ao consumo de bebidas alcoólicas. Observou-se prevalência de consumo de 58,4 (IC95\%: [49,6; 67,2]) entre os homens e de 28,2\% (IC95\%: $[21,8 ; 34,6]$ ) entre as mulheres, sendo a diferença significativa $(\mathrm{P}<0,001)$. Homens e mulheres apresentaram diferença também com relação à forma como consomem a substância. Enquanto entre os homens que consomem bebidas alcoólicas 31,9\% (IC95\%: [23,6; $40,2]$ ) o fazem de maneira excessiva, entre as mulheres este grupo corresponde a 15,3\% (IC95\%: $[8,9 ; 21,79])$, sendo a diferença significativa $(\mathrm{P}<$ $0.001)$.

Em relação às condições de saúde e aos riscos associados ao consumo de bebidas alcoólicas (Tabela 2), os indivíduos que apresentaram consumo excessivo de álcool reportaram melhor autoavaliação em saúde. Entre estes, $48.7 \%$ reportaram a saúde regular/ruim/muito ruim, fração menor que o observado no restante do grupo: $56,3 \%$ entre abstêmios e 56,5\% entre os consumidores moderados. Também foi neste grupo que se observou menor absenteísmo por motivo de saúde $(67,5 \%)$ e menor uso de medicamento. Por outro lado, foi o grupo com maior relato de envolvimento de acidentes de trânsito e episódios de violência física. Quanto à depressão, esta foi menos prevalente entre os que consomem bebidas alcoólicas moderadamente.

A análise bivariada indicou a seguintes variáveis para inclusão no modelo final $(p<0,20)$. A cor da pele autorreferida, pela menor propensão daqueles que se declararam pretos, de ter um consumo moderado $(\mathrm{p}=0.07)$. A escolaridade que apresentou efeito mais intenso entre os que cursaram pelo menos 8 anos de estudos para o consumo moderado, e associação para escolaridade igual ou superior a 4 anos quanto aos consumidores excessivos de álcool. O consumo de bebidas alcoólicas de forma moderada ou excessiva foi maior entre aqueles que estavam em

Tabela 2. Distribuição de frequência do consumo de bebidas alcoólicas moderado e excessivo em relação às condições de saúde e aos riscos associados ao consumo de álcool dos indivíduos. Projeto COMQUISTA, Brasil, 2011.

\begin{tabular}{|c|c|c|c|c|}
\hline \multirow[b]{2}{*}{ Variáveis } & \multicolumn{4}{|c|}{ Consumo de bebidas alcoólicas } \\
\hline & $\begin{array}{c}\text { Amostra total } \\
\mathrm{N}(\%)\end{array}$ & $\begin{array}{r}\text { Abstêmio } \\
\mathrm{N}(\%)\end{array}$ & $\begin{array}{c}\text { Moderado } \\
\text { N (\%) }\end{array}$ & $\begin{array}{c}\text { Excessivo } \\
\mathrm{N}(\%)\end{array}$ \\
\hline \multicolumn{5}{|l|}{ Condições de Saúde } \\
\hline \multicolumn{5}{|l|}{ Autoavaliação de saúde } \\
\hline Muito Boa/Boa & $332(44,4)$ & $191(43,7)$ & $100(43,5)$ & $41(51,3)$ \\
\hline Regular/Ruim/ Muito Ruim & $415(55,6)$ & $246(56,3)$ & $130(56,5)$ & $39(48,7)$ \\
\hline \multicolumn{5}{|l|}{ História de Doença Crônica } \\
\hline Nenhuma & $308(41,1)$ & $168(38,3)$ & $106(45,9)$ & $34(42,5)$ \\
\hline Uma & $267(35,6)$ & $157(35,8)$ & $75(32,5)$ & $35(43,7)$ \\
\hline Duas ou mais & $175(23,3)$ & $114(25,9)$ & $50(21,6)$ & $11(13,8)$ \\
\hline \multicolumn{5}{|c|}{ Deixou de trabalhar por motivo de saúde } \\
\hline Não & $566(75,5)$ & $331(75,4)$ & $181(78,4)$ & $54(67,5)$ \\
\hline Sim & $184(24,5)$ & $108(24,6)$ & $50(21,6)$ & $26(32,5)$ \\
\hline \multicolumn{5}{|l|}{ Depressão } \\
\hline Sem depressão & $607(80,9)$ & $347(79,0)$ & $195(84,4)$ & $65(81,3)$ \\
\hline Deprimido & $143(19,1)$ & $92(21,0)$ & $36(15,6)$ & $15(18,7)$ \\
\hline \multicolumn{5}{|c|}{ Uso de Medicamentos (últimos 15 dias) } \\
\hline Não & $435(58,0)$ & $225(51,3)$ & $151(65,4)$ & $59(73,7)$ \\
\hline Sim & $315(42,0)$ & $214(48,7)$ & $80(34,6)$ & $21(26,3)$ \\
\hline \multicolumn{5}{|l|}{ Riscos associados } \\
\hline \multicolumn{5}{|l|}{ Acidentes de trânsito } \\
\hline Não & $737(98,3)$ & $435(99,1)$ & $227(98,3)$ & $75(93,7)$ \\
\hline Sim & $13(1,7)$ & $4(0,9)$ & $4(1,7)$ & $5(6,3)$ \\
\hline \multicolumn{5}{|l|}{ Vítimas de violência física/agressão } \\
\hline Não & $738(98,4)$ & $433(98,6)$ & $229(99,1)$ & $76(95,0)$ \\
\hline Sim & $12(1,6)$ & $6(1,4)$ & $2(0,9)$ & $4(5,0)$ \\
\hline
\end{tabular}


atividade laborativa. Viver sem companheiro(a) esteve associado com o consumo excessivo ( $\mathrm{p}=$ 0.03). Com relação aos hábitos de vida, os fumantes mostraram maior propensão ao uso de álcool ( $\mathrm{p}<0.001$ ), especialmente o excessivo e o consumo inadequado de frutas e verduras se mostrou associado ao consumo moderado ( $\mathrm{p}=$ $0,12)$. Não se verificou associação entre o consu- mo de bebidas alcoólicas e a prática regular de exercício físico e o indicador de ser quilombola e essas variáveis não foram incluídas no modelo final (Tabela 3).

As associações encontradas no modelo final não se diferenciaram muito daquelas obtidas apenas com o ajuste por idade e sexo. Observou-se que o consumo moderado de álcool foi

Tabela 3. Efeito das características sociodemográficas e de estilo de vida sobre o perfil de consumo de bebidas alcoólicas ajustado por faixa etária e sexo. Projeto COMQUISTA, Brasil, 2011.

\begin{tabular}{|c|c|c|c|c|c|c|}
\hline \multirow{3}{*}{ Variáveis } & \multicolumn{6}{|c|}{ Consumo de bebidas alcoólicas } \\
\hline & \multicolumn{3}{|c|}{ Moderado* $^{*}$} & \multicolumn{3}{|c|}{ Excessivo $^{* *}$} \\
\hline & Or & IC (95\%) & P-valor & Or & IC (95\%) & P-valor \\
\hline \multicolumn{7}{|l|}{ Demográficas } \\
\hline \multicolumn{7}{|l|}{ Idade $^{*}$} \\
\hline $18-24$ & 2,86 & $1,78-4,60$ & $\mathrm{p}<0,001$ & 2,66 & $1,35-5,25$ & 0,005 \\
\hline $25-34$ & 3,76 & $2,80-5,05$ & $\mathrm{p}<0,001$ & 4,64 & $2,39-9,01$ & $\mathrm{p}<0,001$ \\
\hline $35-44$ & 2,30 & $1,79-2,97$ & $\mathrm{p}<0,001$ & 1,88 & $1,25-2,85$ & 0,003 \\
\hline $45-59$ & 1,75 & $1,34-2,29$ & $\mathrm{p}<0,001$ & 2,22 & $1,24-3,98$ & 0,008 \\
\hline $60+$ & 1 & & & 1 & & \\
\hline \multicolumn{7}{|l|}{$\operatorname{Sexo}^{* *}$} \\
\hline Feminino & 1 & & & 1 & & \\
\hline Masculino & 3,31 & $2,76-3,91$ & $\mathrm{p}<0,001$ & 8,95 & $5,78-13,86$ & $\mathrm{p}<0,001$ \\
\hline \multicolumn{7}{|l|}{ Situação Conjugal } \\
\hline Com companheiro(a) & 1 & & & 1 & & \\
\hline Sem companheiro(a) & 1,12 & $0,82-1,52$ & 0,476 & 1,64 & $1,06-2,55$ & 0,027 \\
\hline \multicolumn{7}{|l|}{ Cor da pele autorreferida } \\
\hline Preto & 0,65 & $0,41-1,04$ & 0,073 & 0,86 & $0,55-1,35$ & 0,544 \\
\hline Pardo & 0,76 & $0,49-1,18$ & 0,214 & 1,03 & $0,61-1,73$ & 0,906 \\
\hline Branco e outros & 1 & & & 1 & & \\
\hline \multicolumn{7}{|l|}{ Autodefinição quilombola } \\
\hline Não & 1 & & & 1 & & \\
\hline Sim & 1,24 & $0,73-2,09$ & 0,422 & 0,77 & $0,48-1,25$ & 0,286 \\
\hline \multicolumn{7}{|l|}{ Variáveis socioeconômicas } \\
\hline \multicolumn{7}{|l|}{ Escolaridade } \\
\hline 0 & 1 & & & 1 & & \\
\hline $1-3$ & 1,53 & $1,07-2,19$ & 0,021 & 0,94 & $0,56-1,58$ & 0,816 \\
\hline $4-7$ & 1,51 & $1,10-2,06$ & 0,011 & 1,73 & $1,13-2,64$ & 0,012 \\
\hline 8 e mais & 3,21 & $1,23-8,36$ & 0,017 & 2,85 & $0,81-9,99$ & 0,100 \\
\hline \multicolumn{7}{|l|}{ Trabalha atualmente } \\
\hline Não & 1 & & & 1 & & \\
\hline Sim & 2,04 & $1,60-2,59$ & $\mathrm{p}<0,001$ & 2,75 & $1,51-5,01$ & $\mathrm{P}<0,001$ \\
\hline \multicolumn{7}{|l|}{ Variáveis estilo de vida } \\
\hline \multicolumn{7}{|l|}{ Tabagismo atual } \\
\hline Não & 1 & & & 1 & & \\
\hline Sim & 1,91 & $1,43-2,54$ & $\mathrm{p}<0,001$ & 4,79 & $3,38-6,78$ & $\mathrm{p}<0,001$ \\
\hline \multicolumn{7}{|c|}{ Prática regular de atividade física } \\
\hline Não & 1 & & & 1 & & \\
\hline Sim & 1,11 & $0,93-1,33$ & 0,228 & 1,00 & $0,63-1,60$ & 0,993 \\
\hline \multicolumn{7}{|c|}{ Consumo de verduras/legumes/frutas/suco } \\
\hline 5 vezes ou mais/semana & 1 & & & 1 & & \\
\hline$<5$ vezes/semana & 0,84 & $0,67-1,04$ & 0,120 & 0,93 & $0,50-1,74$ & 0,826 \\
\hline
\end{tabular}

"Variável Idade o efeito de desenho é bruto. " Variável sexo controlado por idade. 
menor entre os que se declararam pretos, mas maior entre as idades 25 a 44 anos, tendo o grupo etário 25-34 apresentado o maior consumo tanto moderado quanto excessivo; os homens, os que se declararam brancos, os que frequentaram a escola, os que estão em atividade laborativa e os fumantes apresentam maior frequência de consumo moderado de álcool. Com relação ao consumo excessivo de álcool, o modelo se difere do anterior com relação à variável cor da pele, que perde a significância, e a escolaridade, que passa a ser significativa a partir dos 8 anos de estudo. Importante ressaltar que foi observado maior consumo de bebidas alcoólicas tanto moderado quanto excessivo entre os indivíduos com escolaridade mais elevada. (Tabela 4 ).

\section{Discussão}

A prevalência de consumo de bebida alcoólica encontrada $(44,0 \%)$ foi comparável a valores de outros estudos realizados no Brasil. O Inquérito domiciliar sobre comportamentos de risco e morbidade referida de doenças e agravos não transmissíveis, realizado em 15 capitais e Distrito Federal, 2002-2003 ${ }^{1}$, considerando a população de 15 anos ou mais, relata prevalência de consumo atual de bebida alcoólica (pelo menos uma dose de bebida alcoólica nos últimos 30 dias) variando entre $32,4 \%$ e $58,6 \%$. Estudo nacional realizado com a população de 18 anos ou mais encontrou prevalência de $52 \%$, sendo $65 \%$ para homens e $41 \%$ para mulheres, para o uso na vida inteira. Estudos de menor abrangência geográfica encontraram prevalências variando entre $44 \%$ e $70 \%{ }^{26-28}$. Deve-se ressaltar que cuidados devem

Tabela 4. Razão das chances de consumo moderado e excessivo de álcool, segundo variáveis sociodemográficas e de estilo de vida. Projeto COMQUISTA, Brasil, 2011.

\begin{tabular}{|c|c|c|c|c|c|c|}
\hline \multirow{3}{*}{ Variáveis } & \multicolumn{6}{|c|}{ Consumo de bebidas alcoólicas } \\
\hline & \multicolumn{3}{|c|}{ Moderado } & \multicolumn{3}{|c|}{ Excessivo } \\
\hline & Or & IC (95\%) & P-valor & Or & IC (95\%) & P-valor \\
\hline \multicolumn{7}{|l|}{ Idade } \\
\hline $18-24$ & 1,58 & $0,93-2,70$ & 0,090 & 1,20 & $0,43-3,32$ & 0,729 \\
\hline $25-34$ & 2,70 & $1,79-4,07$ & $<0,001$ & 3,27 & $1,07-10,04$ & 0,039 \\
\hline $35-44$ & 1,45 & $1,00-2,10$ & 0,051 & 0,97 & $0,42-2,23$ & 0,942 \\
\hline $45-59$ & 1,12 & $0,85-1,46$ & 0,414 & 1,14 & $0,45-2,86$ & 0,784 \\
\hline $60+$ & 1 & & & 1 & & \\
\hline \multicolumn{7}{|l|}{ Sexo } \\
\hline Feminino & 1 & & & 1 & & \\
\hline Masculino & 2,02 & $1,58-2,58$ & $<0,001$ & 3,73 & $2,29-6,08$ & $\mathrm{p}<0,001$ \\
\hline \multicolumn{7}{|l|}{ Situação Conjugal } \\
\hline Com companheiro(a) & 1 & & & 1 & & \\
\hline Sem companheiro $(a)$ & 1,02 & $0,76-1,37$ & 0,901 & 1,53 & $0,92-2,54$ & 0,098 \\
\hline \multicolumn{7}{|l|}{ Cor da pele autorreferida } \\
\hline Preto & 0,63 & $0,41-0,99$ & 0,044 & 0,88 & $0,53-1,47$ & 0,625 \\
\hline Pardo & 0,75 & $0,49-1,14$ & 0,179 & 1,10 & $0,60-2,02$ & 0,752 \\
\hline Branco e outros & 1 & & & 1 & & \\
\hline \multicolumn{7}{|l|}{ Escolaridade } \\
\hline 0 & 1 & & & 1 & & \\
\hline $1-3$ & 1,60 & $1,10-2,34$ & 0,016 & 1,08 & $0,61-1,92$ & 0,780 \\
\hline $4-7$ & 1,61 & $1,17-2,21$ & 0,004 & 2,05 & $1,31-3,22$ & 0,002 \\
\hline 8 e mais & 3,67 & $1,29-10,48$ & 0,016 & 4,34 & $1,14-16,52$ & 0,032 \\
\hline \multicolumn{7}{|l|}{ Trabalha atualmente } \\
\hline Não & 1 & & & 1 & & \\
\hline Sim & 2,05 & $1,62-2,61$ & $<0,001$ & 3,08 & $1,65-5,74$ & 0,001 \\
\hline \multicolumn{7}{|l|}{ Tabagismo atual } \\
\hline Não & 1 & & & 1 & & \\
\hline Sim & 2,13 & $1,66-2,74$ & $<0,001$ & 5,45 & $3,61-8,22$ & $<0,001$ \\
\hline
\end{tabular}

Nota: Razão das chances calculadas considerando como grupo de referência os abstêmios. 
ser tomados ao se comparar esses valores pelas diferenças nas definições, seja do período de referência para avaliação do evento, seja na população de referência.

Foi observado que $10,7 \%$ da amostra apresentaram um padrão de consumo considerado de risco para bebidas alcoólicas, incluindo nesta categoria os que consomem durante a semana uma quantidade excessiva de álcool, sem necessariamente fazer uso exagerado da substância em uma única ocasião, e aqueles que eventualmente utilizam o álcool em quantidade abusiva. Sabe-se que é importante diferenciar os dois tipos de usuários nos estudos que envolvem o uso de álcool, pelas características dos usuários e pelas consequências do comportamento. $\mathrm{O}$ uso contínuo e permanente de álcool está associado ao aumento de doenças crônicas, enquanto o uso excessivo esporádico está relacionado a acidentes e comportamento de risco ${ }^{11,27}$. Esta diferenciação de consumo e suas relações com a saúde e risco não foi possível no nosso estudo. O tamanho reduzido da amostra associado à prevalência do evento levaram a um pequeno número de casos. O consumo excessivo esporádico foi relatado por 76 indivíduos. Entre eles, 21 relataram ser também consumidores da substância em excesso e regularmente. Foram observados apenas 4 relatos de consumo excessivo exclusivamente por uso regular. Com isso, não foi possível, na nossa amostra, em que prevaleceu o consumo excessivo esporádico, diferenciar entre os dois tipos de comportamento.

No Brasil estima-se que $17 \%$ da população das capitais brasileiras fazem uso excessivo esporádico de álcool, sendo que esta porcentagem variou entre $11,9 \%$ e 23,6\% entre as capitais, com Salvador apresentando a maior porcentagem ${ }^{29}$.

Entre as variáveis sociodemográficas estudadas, sexo, idade, escolaridade e situação de emprego apresentaram associações com o consumo excessivo e moderado de álcool, com perfil semelhante de associação para os dois tipos de consumo: jovens consumindo mais que os idosos, com maior consumo na faixa etária de 25 a 34 anos; maior consumo entre os homens, entre os de escolaridade mais elevada e entre aqueles envolvidos em atividade laborativa. Nossos resultados corroboram em parte os resultados da literatura. Tem sido relatado maior uso de álcool entre homens e jovens ${ }^{4,11}$. Segundo Laranjeira et al. ${ }^{25} \mathrm{e}$ Ferraroni et al..$^{30} \mathrm{o}$ álcool é consumido com maior frequência na faixa etária de 18 a 34 anos e entre menores de 55 anos, como encontrado nestes estudos, em que se observou uma tendência de di- minuição do consumo de álcool com o aumento da idade, tanto em homens como em mulheres. A associação com a escolaridade é mais complexa: o consumo moderado é maior entre os indivíduos com maior grau de escolaridade, ocorrendo o inverso para o consumo excessivo.

Karlamangla et al. ${ }^{31}$ encontraram maior risco de consumo de álcool associado a não ter companheiro. Nosso estudo não encontrou associação significativa entre o consumo de álcool e o estado civil. Entretanto, ao analisarmos o consumo excessivo, encontramos uma razão de chances de 1,50 para os que estão sem companheiro, com probabilidade de significância da ordem de $10 \%$. Considerando que o poder da amostra para esta comparação em particular era muito baixo, pode-se considerar que nosso resultado indica na mesma direção do estudo citado. É reconhecido o papel da relação estável na manutenção de hábitos de vida saudáveis e o menor envolvimento com comportamentos de risco para os indivíduos em união estável ${ }^{31}$.

Diferentemente de outros estudos realizados em populações de grandes centros urbanos em que se verificou discreta tendência de prevalências mais altas de consumo de álcool entre pretos, $\operatorname{pardos}^{32}$, e do estudo de âmbito nacional de $2005^{10}$ em que também se observou entre os entrevistados pretos e indígenas maiores propensões de consumo regular de álcool, no nosso estudo os que se declararam pretos ou pardos apresentaram menor prevalência de consumo moderado e não se verificou diferença com relação à cor autorreferida no consumo excessivo. Estudo de base populacional realizado em Salvador-Ba, em 2001, também não encontrou efeito de cor no consumo de álcool ${ }^{12}$.

A literatura internacional já menciona uma menor dependência do álcool entre os negros ao avaliar a associação entre educação e raça com risco de vida e dependência de álcool nos Estados Unidos, no qual foram comparados negros e brancos não hispânicos ${ }^{33}$.

Considerando a escolaridade como marcador de nível socioeconômico, este estudo encontrou gradiente social positivo para o consumo de álcool como já verificado em diversas outras pesquisas. Entretanto, no nosso estudo, este gradiente positivo foi verificado também para o consumo pesado, o que contraria a maior parte dos estu$\operatorname{dos}^{34,35}$. Na análise da associação do consumo do álcool com indicadores sociais, parte-se do entendimento que o consumo moderado é um comportamento ou estilo de vida, enquanto o consumo excessivo é visto como um mau uso da 
substância, mais próximo do abuso ou da dependência que seriam indicadores de morbidade ${ }^{34}, 0$ que justificaria as direções das associações.

Uma possível explicação para os resultados divergentes encontrados na nossa amostra seria a composição da população. A nossa amostra provém de uma população de nível socioeconômico muito baixo, com escolaridade mediana de 3 anos de estudos, sendo que mais de $95 \%$ dos indivíduos não completaram o ensino médio. Os estratos superiores dessa população corresponderiam a estratos medianos de populações mais abrangentes. Bloomfield et al. ${ }^{34}$ em pesquisa sobre uso da álcool na Alemanha observaram relação não linear entre nivel socioeconômico e consumo excessivo de álcool, com as classes sociais intermediárias apresentando o maior consumo. Outros estudos no Brasil encontraram asssociação semelhante à encontrada no nosso estudo ${ }^{12}$.

Os resultados do nosso estudo reforçam as conclusões mencionadas por Guimarães et al. ${ }^{28}$ de que em populações adultas ocorrem associações diretas entre o consumo excessivo de álcool e tabagismo, observadas em outros estudos ${ }^{36,37}$. Esta relação ocorreu para os dois tipos de consumo e não se abranda com o controle pelas demais variáveis; inclusive se intensifica. O tabagismo aumenta a chance de consumo de bebidas alcoólicas, independentemente do tipo de tabaco consumido. Observa-se também que a magnitude da associação entre o consumo de tabaco e de bebidas alcoólicas aumenta com o aumento da frequência do segundo ${ }^{38,39}$.

Algumas limitações metodológicas do presente estudo devem ser apontadas. O desenho transversal do estudo utilizado para a coleta de dados impossibilita estabelecer a natureza da relação entre exposição e evento. Outra potencial limitação do estudo refere-se à autorreferência ao consumo de bebidas alcoólicas que pode ter sido subestima$\mathrm{da}$, principalmente quanto às doses elevadas, na medida em que há restrição social a esse comportamento. Deve-se ainda ressaltar a limitação ad- vinda do instrumento utilizado que, por ser uma adaptação do questionário da Pesquisa Nacional de Saúde, tinha um enfoque mais urbano, não contemplando as especificidades das populações rurais e quilombolas. Percebe-se no questionário da PNS a ausência na avaliação das atividades do setor primário de produção (agricultura, pecuária e extrativismo), êxodo rural, outros hábitos alimentares e culturais e a ausência de avaliação das políticas públicas destinadas às comunidades quilombolas é a execução destes programas.

Diante da escassez de dados nacionais sobre as questões relacionadas ao uso de álcool na população quilombola, este estudo traz informações relevantes para o entendimento dos padrões e fatores relacionados ao consumo álcool em comunidades remanescentes quilombolas. Apesar das limitações citadas, este foi o primeiro estudo de base populacional realizado em comunidades quilombolas do sudoeste da Bahia que avaliou o consumo de álcool e fatores associados.

Estudos identificando os padrões de consumo de bebidas alcoólicas que ajudem na compreensão da magnitude do problema nos diferentes grupos populacionais são necessários para fornecer informações importantes a gestores de saúde, a fim de que se possam desenvolver ações e políticas eficazes de prevenção e de controle do abuso de álcool. Nosso estudo avança um pouco no entendimento do padrão do consumo de álcool nas comunidades quilombolas de Vitória da Conquista e se faz necessário a fim de avaliar periodicamente o consumo abusivo de álcool e tais tendências de consumo.

O padrão que emerge não é muito distinto da população em geral. Em particular, não se observou associação do comportamento de risco com a cor autorreferida ou a autodefinição quilombola. Observou-se alto consumo de bebidas alcoólicas, com padrão de risco para uma parcela considerável da população, especificamente entre os indivíduos do sexo masculino, mais escolarizados e fumantes.

\section{Colaboradores}

LGV Cardoso, APS Melo e CC César contribuíram no desenvolvimento do projeto, revisão de literatura, análise dos dados, redação do artigo e revisão final do artigo. 


\section{Referências}

1. Instituto Nacional de Câncer (Inca). Coordenação de Prevenção e Vigilância. Inquérito domiciliar sobre comportamentos de risco e morbidade referida de doenças e agravos não transmissíveis. Rio de Janeiro: Inca; 2004.

2. Zakhari S. Alcohol and the Cardiovascular System. Alcohol Health and Research World 1997; 21(1):21-29.

3. Costa JSD, Silveira MF, Gazalle FK, Oliveira SS, Hallal PC, Menezes AMB, Gigante DP, Olinto MTA, Macedo S. Consumo abusivo de álcool e fatores associados: estudo de base populacional. Rev Saude Publica 2004; 38(2):284-291.

4. World Health Organization (WHO). Global status report on alcohol. Geneva: WHO; 2011.

5. Barros MBA, Marín-León L, Oliveira HB, Dalgalarrondo P, Botega NJ. Perfil do consumo de bebidas alcoólicas: diferenças sociais e demográficas no Município de Campinas, Estado de São Paulo, Brasil, 2003. Epidemiol. Serv. Saúde 2008; 17(4):259-270.

6. Gallassi AD, Alvarenga PG, Andrade AG, Couttolenc BF. Custos dos problemas causados pelo abuso do álcool. Rev Psiq Clín 2008; 35(Supl. 1):25-30.

7. Hagger MS, Lonsdale AD, Hein V, Koka A, Lintunen T, Pasi H, Lindwall M, Rudolfsson L, Chatzisarantis NLD. Predicting alcohol consumption and binge drinking in company employees: An application of planned behaviour and self-determination theories. Br J Health Psychol 2012; 17(2):379-407.

8. World Health Organization (WHO). Social determinants of health: the solid facts. $2^{\mathrm{a}}$ ed. Copenhagen: WHO; 2000.

9. Jomar RT, Abreu AMM, Griep RH. Padrões de consumo de álcool e fatores associados entre adultos usuários de serviço de atenção básica do Rio de Janeiro, RJ, Brasil. Cien Saude Colet 2014; 19(1):27-38.

10. Bastos FI, Bertoni N, Hacker MA. Drug and alcohol use: main findings of a national survey, Brazil 2005. Rev Saude Publica 2008; 42(Supl. 1):109-117.

11. Guedes RC, Freitas APF, Vasconcelos TC, Cerqueira GS, Rocha NFM, Pinto RH. Consumo de Álcool em uma Comunidade do Cariri Cearense. Revista Intertox de Toxicologia, Risco Ambiental e Sociedade 2010; 3(3):2643.

12. Almeida-Filho N, Lessa I, Magalhães L, Araújo MJ, Aquino E, James SA, Kawachi I. Social inequality and alcohol consumption-abuse in Bahia, Brazil Interactions of gender, ethnicity and social class. Soc Psychiatry Psychiatr Epidemiol 2005; 40(3):214-222.

13. Batista LE. Masculinidade, raça/cor e saúde. Cien Saude Colet 2005; 10(1):71-80.

14. Araújo EM, Costa MCN, Hogan VK, Araújo TM, Dias $\mathrm{AB}$, Oliveira LOA. A utilização da variável raça/cor em Saúde Pública: possibilidades e limites. Interface (Botucatu) 2009; 13(31):383-394.

15. Brasil. Portaria no 992/GM de 13 de maio de 2009. Política Nacional de Saúde Integral da População Negra. Diário Oficial da União, 2009, 15 mai.

16. Bastos JL, Celeste RK, Faerstein E, Barros AJ. Racial discrimination and health: A systematic review of scales with a focus on their psychometric properties. Soc Scie Med 2010; 70(7):1091-1099.
17. Batista LE, Volochko A, Ferreira CEC, Martins V. Mortalidade da população negra adulta no Brasil. In: Fundação Nacional de Saúde (Funasa): Saúde da população negra no Brasil: contribuições para a promoção da equidade. Brasília: Funasa; 2005. p. 237-314.

18. Lopes F. Mulheres negras e não negras vivendo com HIV/ Aids no estado de São Paulo: um estudo sobre suas vulnerabilidades [tese]. São Paulo: Universidade de São Paulo; 2003.

19. Souza MLP, Garnelo L. Desconstruindo o alcoolismo: notas a partir da construção do objeto de pesquisa no contexto indígena. Rev. Latinoam. Psicopat. Fund. 2006; IX(2):279-292.

20. Ajzen I. The Theory of Planned Behavior. Organizational behavior and human decision processes 1991; 50:179-211.

21. Amorim IG, Germani GI. Quilombos da Bahia: presença incontestável. In: Anais do X Encontro de Geógrafos da América Latina. São Paulo: USP; 2005. p. 1-17.

22. Oliveira e Silva D, Guerrero AFH, Guerrero CH, Toledo LM. A rede de causalidade da insegurança alimentar e nutricional de comunidades quilombolas com a construção da rodovia BR-163, Pará, Brasil. Rev Nutrição 2008; 21 (Supl.):83-97.

23. Bezerra VM, Medeiros DS, Gomes KO, Souzas R, Giatti L, Guimarães MDC. Inquérito de Saúde em Comunidades Quilombolas de Vitória da Conquista/BA (Projeto COMQUISTA): aspectos metodológicos e análise descritiva. Cien Saude Colet 2013; 19(6):1835-1847.

24. Pesquisa Nacional de Saúde (PSN). Inquérito Região Integrada do Distrito Federal (RIDE/DF) [site da internet] 2011 [acessado 2011 abr 1]; [cerca de 105p.] Disponível em: http://www.pns.icict.fiocruz.br.

25. Laranjeira R, Pinsky I, Zaleski M, Caetano R, Duarte PCAV. I Levantamento Nacional sobre os padrões de consumo de álcool na população brasileira. Brasília: Secretaria Nacional Antidrogas; 2007.

26. Bortoluzzi MC, Traebert J, Loguercio A, Kehrig RT. Prevalência e perfil dos usuários de álcool de população adulta em cidade do sul do Brasil. Cien Saude Colet 2010; 15(3):679-685.

27. Ferreira LN, Sales ZN, Casotti CA, Bispo Júnior JP, Braga Júnior ACR. Perfil do consumo de bebidas alcoólicas e fatores associados em um município do Nordeste do Brasil. Cad Saude Publica 2011; 27(8):1473-1486.

28. Guimarães VV, Florindo AA, Stopa SR, César CLG, Barros MBA, Carandina L, Goldbaum M. Consumo abusivo e dependência de álcool em população adulta no Estado de São Paulo, Brasil. Rev. bras. epidemiol. 2010; 13(2):314-325.

29. Brasil. Ministério da Saúde (MS). Secretaria de Vigilância em Saúde. Vigitel Brasil 2011: Vigilância de fatores de risco e proteção para doenças crônicas por inquérito telefônico. Brasília: MS; 2012.

30. Ferraroni M, Decarli A, Franceschi S, La Vecchia C, Enard L, Negri E, Parpinel M, Salvini S. Validity and Reproducibility of Alcohol Consumption in Italy. Int J Epidemiol 1996; 25(4):775-782. 
31. Karlamangla A, Zhou K, Reuben D, Greendale G, Moore A. Longitudinal trajectories of heavy drinking in adults in the United States of America. Addiction 2006; 101(1):91-99.

32. São Paulo. Secretaria Municipal da Saúde. Coordenação de Epidemiologia e Informação - CEInfo. Boletim ISA - Capital 2008 São Paulo (SP), 5: Transtornos Mentais Comuns. Tabagismo. Consumo de Bebida Alcoólica. São Paulo: CEInfo; 2011.

33. Gilman SE, Breslau J, Conron KJ, Koenen KC, Subramanian SV, Zaslavsky AM. Education and race-ethnicity differences in the lifetime risk of alcohol dependence. J Epidemiol Community Health 2008; 62(3):224-230.

34. Bloomfield K, Augustin R, Kraus L. Social Inequalities in Alcohol Use and Misuse in the German General Population. Zeitschrift fur Gesundheitswissenschaften 2000; 8(3):230-242.

35. Van Oers J, Bongers I, Van de Goor L, Garretsen, H. Alcohol consumption, alcohol-related problems, problem drinking, and socioeconomic status. Alcohol and Alcoholism 1999; 34(1):78-88.

36. Vargas D, Oliveira MAF, Araújo EC. Prevalência de dependência alcoólica em serviços de atenção primária à saúde de Bebedouro, São Paulo, Brasil. Cad Saude Publica 2009; 25(8):1711-1720.

37. Herbeth B, Samara A, Stathopoulou M, Siest G, Visvikis-Siest S. Alcohol Consumption, Beverage Preference, and Diet in Middle-Aged Men from the STANISLAS Study. J Nutr Metab [periódico na internet] 2012 [acessado 2013 nov 28];1:[6 p.]. Disponível em: http://www. hindawi.com/journals/jnme/2012/987243/

38. Padrão P, Silva-Matos C, Damasceno A, Lunet L. Association between tobacco consumption and alcohol, vegetable and fruit intake across urban and rural areas in Mozambique. J Epidemiol Community Health 2011; 65(5):445-453.

39. Accortt N, Waterbor J, Beall C, Howard G, Brooks CM. Need to educate primary caregivers about the risk factor profile of smokeless tobacco users. J Cancer Educ 2005; 20(4):222-228.

Artigo apresentado em 28/08/2014

Aprovado em 30/08/2014

Versão final apresentada em 02/08/2014 